\title{
A LI-TYPE CRITERION FOR ZERO-FREE HALF-PLANES OF RIEMANN'S ZETA FUNCTION
}

\author{
PEDRO FREITAS
}

\begin{abstract}
We define a sequence of real functions which coincide with Li's coefficients at one and which allow us to extend Li's criterion for the Riemann Hypothesis to yield a necessary and sufficient condition for the existence of zero-free strips inside the critical strip $0<\Re(z)<1$. We study some of the properties of these functions, including their oscillatory behaviour.
\end{abstract}

\section{INTRODUCTION}

In 1997 Li gave a necessary and sufficient condition for the Riemann Hypothesis $(\mathrm{RH})$ to hold, based on the positivity of a sequence of real numbers $\mathrm{L}$. Li's coefficients may be written as

$$
\lambda_{n+1}=\frac{1}{n !} \frac{d^{n+1}}{d s^{n+1}}\left[s^{n} \log (\xi(s))\right]_{s=1}, \quad n=0,1, \ldots,
$$

and the criterion then states that the RH holds if and only if $\lambda_{n}$ is greater than or equal to zero for all $n=1,2, \ldots$. Here $\xi$ is the function defined by

$$
\xi(s)=s(s-1) \pi^{-s / 2} \Gamma(s / 2) \zeta(s),
$$

where $\Gamma$ and $\zeta$ are, respectively, Euler's gamma and Riemann's zeta functions. As is well known, $\xi$ is an entire function satisfying the functional equation $\xi(s)=\xi(1-s)$.

Equivalently, these coefficients may also be defined as the coefficients in the Taylor series of the function

$$
\psi(z)=\frac{\varphi^{\prime}(z)}{\varphi(z)}=\sum_{n=0}^{\infty} \lambda_{n+1} z^{n}
$$

where $\varphi$ is given by

$$
\varphi(z)=\xi\left(\frac{1}{1-z}\right)
$$

In fact, and as remarked in $\overline{B L}$, the idea behind Li's criterion is that the $\mathrm{RH}$ holds if and only if the coefficients $\lambda_{n}$ satisfy a growth condition, namely, that $\lim \sup \left|\lambda_{n+1}\right|^{1 / n} \leq 1$, and that this may in fact be replaced by the (one-sided) condition on their positivity. From this it follows that the positivity of Li's coefficients is related to the derivatives of the functions $\xi$ and $\varphi$.

At this point it should be mentioned that a set of coefficients which differ from Li's only by a (positive) multiplicative constant was introduced earlier by Keiper

Date: November 11, 2018.

1991 Mathematics Subject Classification. Primary 11M26; Secondary 11M06.

Key words and phrases. Riemann Hypothesis, Li's criterion. 
in [K]. In particular, it is already stated in [K] that the positivity of these coefficients is implied by the RH. I am indebted to André Voros for pointing this out to me.

Li's coefficients have recently been the subject of much study, from both theoretical and numerical points of view - see B, BL, C1, C2, L, M1, M2, V], for instance. As was pointed out in $\mathrm{BL}$, this criterion is not specific for the function $\xi$, and will work for any function which can be written as a product of the form

$$
f(z)=\prod_{\rho \in Z}\left(1-\frac{z}{\rho}\right),
$$

or also in the more general case of a multi-set, provided some conditions are imposed on the asymptotic behaviour of the zero set $Z$. The main purpose of the present note is to show that Li's criterion may be extended in a simple and natural way to provide a necessary and sufficient condition for the existence of a zero-free halfplane $\Re(z)>\tau / 2$, and to study the behaviour of the functions that arise naturally while carrying out this extension. We remark that an extension of Li's criterion to provide zero-free regions for Riemann's zeta function has been considered in $[\mathrm{B}$. However, this uses a completely different approach based on an analysis of the implications of the non-negativity of the first $N$ of Li's coefficients.

Although most of the results presented here apply to a more general function $f$ as above, we will concentrate on the case where $f=\xi$. In this case, and for $\tau$ in $(1,2)$, the condition that there are no zeros of zeta with $\Re(z)>\tau / 2$ is sometimes referred to as the quasi- $\mathrm{RH}$, and we remark that this weaker version of the $\mathrm{RH}$ also remains an open problem. More precisely, it is not known if there exists $\tau_{0}$ on $(1 / 2,1)$ such that there are no zeros in the strip $\Re(s)>\tau_{0}$.

In order to extend Li's criterion, let us define the coefficients

$$
\alpha_{n+1}(\tau)=\left.\frac{1}{n !} \frac{d^{n+1}}{d s^{n+1}}\left[s^{n} \log (\xi(s))\right]\right|_{s=\tau}, n=0,1, \ldots,
$$

where $\tau$ is a positive number. We remark that of the original (implicit) definitions of the coefficients $\lambda_{n}$ in $\underline{\mathrm{L}}$, not all correspond to the expression above. More precisely, apart from equations (1.1) and (1.2), the expression

$$
\sum_{\rho \in Z}\left[1-\left(1-\frac{1}{\rho}\right)^{n}\right]
$$

is also used in that paper as corresponding to $\lambda_{n}$, while in fact this would correspond to $\lambda_{-n}-$ see $\mathrm{BL}$ and also Section 3 below. In fact, at $\tau=1$ it is indeed true that $\lambda_{n}=\lambda_{-n}$, and thus this distinction was not important in Li's paper. However, since this will not be the case for other values of $\tau$, amd we use (1.5) as a starting point, there is a discrepancy between the notation in our paper and that used in other papers, such as $\mathrm{BL}$.

Clearly these functions are analytic for real values of $\tau$, and have singularities at the zeros of the function $\xi$. The main idea here is that while for $\tau$ equal to one we recover Li's coefficients, the positivity of the $\alpha_{n}$ 's for other values of $\tau$ is equivalent to the non-existence of zeros in the half-plane $\Re(s)>\tau / 2$. In particular, and since for $\tau$ smaller than one we obtain a region which contains the critical line, in this case some of the coefficients $\alpha_{n}$ must take on negative values. On the other hand, for $\tau$ greater than or equal to two we are outside the critical strip and hence the $\alpha_{n}$ 's must all be non-negative. 
More precisely, we have the following

Theorem 1. Given $\tau$ on $[1 / 2, \infty)$, the half-plane $\Re(s)>\tau / 2$ is a zero-free region for the Riemann zeta function if and only if $\alpha_{n}(\tau)$ is nonnegative for all positive integer $n$.

Note that Theorem 1 in $\mathrm{BL}$ already allows us to obtain a condition which will ensure that the zeros of the function $\xi$ are confined to a half-plane - this may be done simply by replacing $\rho$ by $\rho / \tau$ in the relevant expressions. The main contribution of the above result is to relate this to the positivity of the coefficients $\alpha_{n}$ defined by (1.5). The proof also shows that these functions are directly related to the Taylor coefficients of the function $\psi$ around the point $z=1-1 / \tau$.

Since we will follow an approach similar to that used in Li's paper, in order to prove Theorem 1 we will need some results concerning Taylor expansions of the function $\varphi(z)=\xi(1 /(1-z))$. This will be done in Section 2 where the behaviour of the corresponding Taylor coefficients is analysed. We shall present some simpler proofs for some known results, and also obtain some new monotonicity properties which we believe to be of interest in their own right.

In Section [3] we prove Theorem [1] and in Section 4 we obtain some further properties of the coefficients $\alpha_{n}$. These include a system of differential equations satisfied by the $\alpha_{n}$ 's - see Theorem 4.3 - and some oscillatory properties which are summarized in the following

Theorem 2. For $n=1,2, \ldots$ and $0 \leq \tau<2$ the following facts hold

(i) $\alpha_{n}(0)=n \frac{\xi^{\prime}(0)}{\xi(0)}<0$;

(ii) there exists a sequence $1 / 2=a_{1}>a_{2}>\ldots$ such that $\alpha_{n}\left(a_{n}\right)=0$ and $\alpha_{n}(\tau)<0$ for $\tau<a_{n}$

(iii) between any two real zeros of $\alpha_{n}(\tau)$ there exists at least one real zero of $\alpha_{n+1}(\tau)$

(iv) for any positive integer $N$ there exists a positive integer $n_{0}$ such that the function $\alpha_{n}(\tau)$ has at least $N$ zeros on the interval $(0,2)$, for all $n$ larger than $n_{0}$.

We then proceed to extend the definition of the coefficients $\alpha_{n}$ to the case of real $n$. This is done in Section [5] where some alternative representations for this more general function are given. Finally, in the last section, we briefly discuss the results obtained.

\section{Some Properties of the TAYlor COEFficients of the FUNCTIONS $\varphi$ AND $\xi$}

We begin by proving a simple lemma which will be useful in the sequel. This can easilly be obtained from known results (see $[\mathbf{K}$, for instance), but we include it here in a form appropriate for our purposes.

Lemma 2.1. Let $f$ be an analytic function in an open subset $\Omega$ of $\mathbb{C}$, and let $z_{0}<z_{1}$ be two (real) points in $\Omega$. Write

$$
f(z)=\sum_{n=0}^{\infty} a_{n}\left(z-z_{0}\right)^{n}=\sum_{n=0}^{\infty} b_{n}\left(z-z_{1}\right)^{n},
$$

and assume the following conditions hold 
(C1) $a_{n}$ is real and non-negative for $n=0,1, \ldots$

(C2) the first series is convergent at $z=z_{1}$

Then $b_{n} \geq 0$ for $n=0,1, \ldots$. Furthermore, if $a_{m}>0$ for some integer $m$, then $b_{n}>0$ for $n=0, \ldots, m$.

Proof. From

$$
f(z)=\sum_{n=0}^{\infty} a_{n}\left(z-z_{0}\right)^{n}=\sum_{n=0}^{\infty} b_{n}\left(z-z_{1}\right)^{n}
$$

and taking $p$ derivatives with respect to $z$, we have that

$$
\sum_{n=p}^{\infty}\left(\begin{array}{c}
n \\
p
\end{array}\right) a_{n}\left(z-z_{0}\right)^{n-p}=\sum_{n=p}^{\infty}\left(\begin{array}{c}
n \\
p
\end{array}\right) b_{n}\left(z-z_{1}\right)^{n-p} .
$$

Making now $z=z_{1}$ yields

$$
b_{p}=\sum_{n=p}^{\infty}\left(\begin{array}{c}
n \\
p
\end{array}\right) a_{n}\left(z_{1}-z_{0}\right)^{n-p},
$$

from which the result follows.

Using this we obtain a simple proof of the following result regarding the sign of the derivatives of the function $\xi$, which can already be found in C1. This is a generalization of a result of Pustyl'nikov's, who proved in $[\mathrm{P}$ that a necessary condition for the $\mathrm{RH}$ to hold is that all even derivatives of $\xi$ at the point $z=1 / 2$ are positive. While these proofs are based on the integral representation of the function $\pi^{-z / 2} \Gamma(z / 2) \zeta(z)$, here we show this by direct series manipulation and the result at $z=1 / 2$, which may already be found on page 41 of $[\mathrm{E}$.

Theorem 2.2. Even derivatives of $\xi$ are positive for all real values of $z$, while odd derivatives are positive for $z>1 / 2$ and negative for $z<1 / 2$.

Proof. Consider the series development of $\xi$ at a point $z_{0}$, that is,

$$
\xi(z)=\sum_{n=0}^{\infty} s_{n}\left(z_{0}\right)\left(z-z_{0}\right)^{n} .
$$

Since $\xi$ is an entire function,

$$
s_{0}(1 / 2)=\xi(1 / 2)=-\Gamma(1 / 4) \zeta(1 / 2) /\left(4 \pi^{1 / 4}\right) \approx .994242,
$$

and we have that $s_{2 n}(1 / 2)$ is strictly positive, it follows from the lemma that $s_{n}\left(z_{0}\right)$ is strictly positive for all $n$ and all real $z$ larger than $1 / 2$. The result for $z$ less than $1 / 2$ now follows by repeated differentiation of the functional equation for $\xi$.

Write now the series development for the function $\varphi$ around a real number $z_{0}$ as

$$
\varphi(z)=\sum_{n=0}^{\infty} c_{n}\left(z_{0}\right)\left(z-z_{0}\right)^{n}
$$

This series is well defined for all $z_{0}$ different from one, and its radius of convergence is equal to $\left|1-z_{0}\right|$.

We shall first show that the coefficients $c_{n}(-1)$ are all positive with the exception of $c_{1}(-1)$ which vanishes - in what follows, and provided there is no room for confusion, we shall drop the explicit reference to the point $z_{0}$. 
Theorem 2.3. For all positive integers $n$ different from 1 , we have that $\varphi^{(n)}(-1)>$ 0 , while $\varphi^{\prime}(-1)=0$.

Proof. From the definition of $\varphi$ we see that $\varphi(-1)=\xi(1 / 2)>0$. Differentiating both sides of (1.3) with respect to $z$ yields

$$
\varphi^{\prime}(z)=\xi^{\prime}\left(\frac{1}{1-z}\right)\left(\frac{1}{1-z}\right)^{2} .
$$

At $z=-1$ this gives $\varphi^{\prime}(-1)=0$, while further repeated differentiation of this identity will now yield that the $k^{\text {th }}$ derivative of $\varphi$ at -1 is a linear combination with positive coefficients of derivatives of $\xi$ taken at $1 / 2$. Since for $k$ larger than 1 there will always exist at least one even derivative of $\xi$, the result follows.

An immediate consequence of this result is the positivity of the coefficients in the series development of $\varphi$ around any point on the interval $(-1,1)$.

Corollary 2.4. For all $z$ in $(-1,1), c_{n}(z)>0$.

Proof. It follows by direct application of Lemma 2.1 with $z_{0}=-1$, taking into account that $\varphi$ 's only singularity is at $z=1$, and thus condition $(\mathrm{C} 2)$ in the lemma is satisfied, while $(\mathrm{C} 1)$ is a direct consequence of Theorem 2.3

Remark 2.5. The coefficients $c_{n}(0)$ correspond to the coefficients $a_{n}$ in Li's paper.

Remark 2.6. This may also be proven in the same way as in Theorem [2.3] using the positivity results of Theorem 2.2

As a consequence of the positivity of the coefficients $c_{n}$ we see that they are actually increasing functions of $z_{0}$.

Corollary 2.7. The functions $c_{n}:(-1,1) \rightarrow \mathbb{R}^{+}$are strictly monotonically increasing.

Proof. Since

$$
c_{n}(z)=\frac{1}{n !} \varphi^{(n)}(z)
$$

it follows that $c_{n}^{\prime}(z)=(n+1) c_{n+1}(z)>0$

Furthermore, it is possible to show that for $z_{0}$ on $[0,1)$, these coefficients are also increasing functions of $n$, while for $z_{0}$ on $[-1,-1 / 2)$ they will be decreasing.

Theorem 2.8. We have that

$$
c_{n}\left(z_{0}\right)-c_{n-1}\left(z_{0}\right) \begin{cases}>0, & z_{0} \in[0,1) \\ <0, & z_{0} \in[-1,-1 / 2]\end{cases}
$$

for $n=2,3, \ldots$

Proof. Let

$$
\theta(z)=\left[1-\left(z-z_{0}\right)\right] \xi\left(\frac{1}{1-z}\right)=c_{0}+\sum_{n=1}^{\infty}\left(c_{n}-c_{n-1}\right)\left(z-z_{0}\right)^{n} .
$$

and differentiate $\theta$ twice with respect to $z$ to obtain

$$
\theta^{\prime \prime}(z)=\frac{1+2 z_{0}}{(1-z)^{3}} \xi^{\prime}\left(\frac{1}{1-z}\right)+\frac{z_{0}}{(1-z)^{4}} \xi^{\prime \prime}\left(\frac{1}{1-z}\right)
$$


From this we see that $\theta^{\prime \prime}\left(z_{0}\right)$ is positive for $z_{0} \in[0,1)$ and negative for $z_{0} \in$ $[-1,-1 / 2]$. Further differentiation with respect to $z$ gives rise to a sum of terms which are derivatives of $\xi$ multiplied by constants which are either all positive or all negative, depending on whether $z_{0} \in[0,1)$ or $z_{0} \in[-1,-1 / 2]$, respectively. Since in either case $1 /\left(1-z_{0}\right)$ is greater than or equal to $1 / 2$, the derivatives of $\xi$ are non-negative and at least one will be positive.

The result now follows, as $c_{n}-c_{n-1}=\theta^{(n)}\left(z_{0}\right)$ for $n=2,3, \ldots$.

Since the radius of convergence of the series for $\varphi$ around $z_{0}$ is $\left|1-z_{0}\right|$, it follows that, for negative $z_{0}, c_{n}\left(z_{0}\right) \rightarrow 0$ as $n$ goes to infinity. We shall now prove that for all $z_{0}$ on $[0,1)$ these coefficients must go to infinity as $n$ goes to infinity.

Theorem 2.9. For $z_{0} \in[0,1)$ we have that

$$
\lim _{n \rightarrow \infty} c_{n}\left(z_{0}\right)=\infty
$$

Proof. From the expansion for $\xi$ given by (2.1) it follows that

$$
\begin{aligned}
\varphi(z) & =\sum_{n=0}^{\infty} s_{n}(1)\left(\frac{z}{1-z}\right)^{n} \\
& =s_{0}(1)+\sum_{n=0}^{\infty}\left[\sum_{k=0}^{n}\left(\begin{array}{c}
n \\
k
\end{array}\right) s_{k+1}(1)\right] z^{n+1} .
\end{aligned}
$$

This yields

$$
c_{n+1}(0)=\sum_{k=0}^{n}\left(\begin{array}{l}
n \\
k
\end{array}\right) s_{k+1}(1) \geq s_{1}(1)+n \sum_{k=1}^{n} \frac{1}{k !} s_{k+1}(1),
$$

from which the result follows, since the $s_{k}$ 's are positive and, by Corollary 2.7 the coefficients $c_{n}$ are increasing with $z$.

\section{Proof of Theorem 1}

We shall now turn to the series for the function $\psi$ and the proof of Theorem 1 . In order to do this, we begin by establishing the equivalence of the different formulations for the functions $\alpha_{n}(\tau)$.

Lemma 3.1. For each positive integer $n$ and all real $\tau$ the functions $\alpha_{n}(\tau)$ defined by 1.5) satisfy the following relations:

(i)

$$
\alpha_{n}(\tau)=\frac{1}{\tau} \sum_{\rho}\left[1-\left(\frac{\rho}{\rho-\tau}\right)^{n}\right],
$$

where $\rho$ runs over the nontrivial zeros of the zeta function and the terms corresponding to $\rho$ and $1-\rho$ are paired together.

(ii) Define the power series coefficients $d_{n}\left(z_{0}\right)$ by

$$
\psi(z)=\frac{\varphi^{\prime}(z)}{\varphi(z)}=\sum_{n=0}^{\infty} d_{n}\left(z_{0}\right)\left(z-z_{0}\right)^{n} .
$$

Then

$$
\alpha_{n}(\tau)=\frac{1}{\tau^{n+1}} d_{n-1}\left(1-\frac{1}{\tau}\right)(\tau \neq 0)
$$


Proof. Starting from the product representation of $\xi$, that is,

$$
\xi(s)=\prod_{\rho}\left(1-\frac{s}{\rho}\right)
$$

where, as usual, the product runs over the nontrivial zeros of the zeta function and each term is paired with that corresponding to $1-\rho$, we obtain that

$$
\varphi(s)=\prod_{\rho}\left(\frac{1-1 / \rho-s}{1-s}\right) .
$$

By taking the logarithmic derivative, we then have that

$$
\begin{aligned}
\psi(s) & =-\sum_{\rho}\left(\frac{1}{\rho-1-\rho s} \frac{1}{1-s}\right) \\
& =-\sum_{\rho} \frac{1}{\rho\left(1-z_{0}\right)-1} \frac{1}{1-\frac{\rho\left(s-z_{0}\right)}{\rho\left(1-z_{0}\right)-1}} \frac{1}{1-z_{0}} \frac{1}{1-\frac{s-z_{0}}{1-z_{0}}} \\
& =\sum_{n=0}^{\infty} \tau^{n+1} \sum_{\rho}\left[1-\left(\frac{\rho}{\rho-\tau}\right)^{n+1}\right]\left(s-z_{0}\right)^{n},
\end{aligned}
$$

where $z_{0}$ is any real number on $[-1,1)$ and $\tau=1 /\left(1-z_{0}\right)$. We thus obtain that

$$
d_{n}\left(z_{0}\right)=\tau^{n+1} \sum_{\rho}\left[1-\left(\frac{\rho}{\rho-\tau}\right)^{n+1}\right]
$$

On the other hand, we have that

$$
\begin{aligned}
\alpha_{n+1}(\tau) & =\frac{1}{n !} \frac{d^{n+1}}{d s^{n+1}}\left[s^{n} \log (\xi(s))\right]_{s=\tau} \\
& =-\frac{1}{\tau} \sum_{\rho} \sum_{k=0}^{n}\left(\begin{array}{c}
n+1 \\
k
\end{array}\right)\left(\frac{\rho}{\tau}-1\right)^{k-n-1} \\
& =\frac{1}{\tau} \sum_{\rho}\left[1-\left(\frac{\rho}{\rho-\tau}\right)^{n+1}\right] .
\end{aligned}
$$

which proves $(i)$, and (ii) now follows by comparing this with the expression obtained for $d_{n}\left(z_{0}\right)$.

To prove Theorem 11 we begin by noticing that equation (3.1) yields that the coefficients $d_{n}\left(z_{0}\right)$ will be non-negative if

$$
\left|\frac{\rho}{\rho-\tau}\right| \leq 1
$$

Hence, if all zeros have real part smaller than or equal to $\tau / 2$, part ( $i i)$ of Lemma 3.1 implies that the coefficients $\alpha_{n}(\tau)$ will be non-negative.

Proceeding in the same fashion as in Li's paper, we shall now obtain a recurrence relation between the coefficients $c_{n}$ and $d_{n}$ at a point $z_{0}$ on $[-1,1)$ - note that since 
$\varphi$ is positive on this interval, $\psi$ is analytic in a neighbourhood of any point on the same interval. More precisely, from the definition of $\psi$ we have that

$$
\sum_{n=1}^{\infty} n c_{n}\left(z-z_{0}\right)^{n-1}=\left(\sum_{n=0}^{\infty} c_{n}\left(z-z_{0}\right)^{n}\right)\left(\sum_{n=0}^{\infty} d_{n}\left(z-z_{0}\right)^{n}\right),
$$

yielding the relations

$$
d_{n-1}=n \frac{c_{n}}{c_{0}}-\frac{1}{c_{0}} \sum_{k=1}^{n-1} c_{k} d_{n-k-1}, \quad n=1,2, \ldots
$$

From Corollary 2.4 we know that the coefficients $c_{n}$ are all positive. Still following the steps of Li's proof, assume now that the coefficients $d_{n}$ are all non-negative. Then, $d_{n-1} \leq n c_{n} / c_{0}$, and

$$
\sum_{n=1}^{\infty}\left|d_{n-1}\left(z-z_{0}\right)^{n-1}\right| \leq \frac{1}{c_{0}} \sum_{n=1}^{\infty} n c_{n}\left|z-z_{0}\right|^{n-1}=\frac{1}{c_{0}} \varphi^{\prime}\left(z_{0}+\left|z-z_{0}\right|\right) .
$$

It follows that the series

$$
\sum_{n=0}^{\infty}\left|d_{n}\left(z-z_{0}\right)^{n}\right|
$$

converges for all $\left|z-z_{0}\right|<1-z_{0}$. This finishes the proof of Theorem 1

\section{Some Properties of the COEFFicients $\alpha_{n}$}

We shall now study the behaviour of the functions $\alpha_{n}$. Although Theorem 1only considers the case of $\tau$ larger than $1 / 2$, we will consider the whole of $\mathbb{R}^{+}$unless it is explicitly stated otherwise. We shall stick to the notation used in the previous section.

4.1. A relaxed sufficient condition. We begin by giving a result in the spirit of Corollary 1 in [BL], namely, we show that the sufficient condition of Theorem 1 can be somewhat relaxed.

Proposition 4.1. Let

$$
g(z)=\sum_{n=0}^{\infty} \gamma_{n}\left(z-z_{0}\right)^{n}
$$

be any function such that the coefficients $\gamma_{n}=\gamma_{n}(\tau)$ are all nonnegative and such that the Taylor series for $g$ around $z_{0}$ has radius of convergence at least $1-z_{0}$. Then, given $\tau$ on $[1 / 2, \infty)$, the half-plane $\Re(s)>\tau / 2$ is a zero-free region for the Riemann zeta function if and only if $\alpha_{n}(\tau)+\gamma_{n-1}(\tau)$ is nonnegative for all positive integer $n$.

Proof. That the RH implies $\alpha_{n}(\tau)+\gamma_{n-1}(\tau) \geq 0$ is a trivial consequence of Theorem 1 and the hypothesis that $\gamma_{n} \geq 0$ for all $n$.

To prove the implication in the other direction we consider the auxiliary function

$$
h(z)=e^{\int_{0}^{z} g(t) d t} \varphi(z) .
$$

We have that the Taylor series for $h$ around a point $z_{0}$ on $[-1,1)$ will also have radius of convergence $1-z_{0}$, and the corresponding Taylor coefficients are also 
nonnegative. It folows that

$$
\begin{aligned}
H(z) & :=\frac{h^{\prime}(z)}{h(z)} \\
& =\psi(z)+g(z) \\
& =\sum_{n=0}^{\infty}\left[d_{n}\left(z_{0}\right)+\gamma_{n}\left(z_{0}\right)\right]\left(z-z_{0}\right)^{n} .
\end{aligned}
$$

Since the radius of convergence of the series for $g$ is $1-z_{0}$, it follows that the radius of convergence for the Taylor series of $H$ around $z_{0}$ will still be that of the series for $\psi$. Proceeding now as in the second part of the proof of Theorem 1 with $d_{n}\left(z_{0}\right)+\gamma_{n}\left(z_{0}\right)$ in place of $d_{n}\left(z_{0}\right)$ we obtain the desired result.

A straightforward consequence of this result is that for a value of $\tau$ larger than $1 / 2$ for which the half-plane $\Re(s)>\tau / 2$ is not a zero-free region of the zeta function there cannot exist only a finite number of the $\alpha_{n}$ 's which are negative.

Corollary 4.2. If the half-plane $\Re(s)>\tau_{0} / 2$ is not a zero-free region of the Riemann zeta function for some $\tau_{0}$ on $[1 / 2,2)$, then there exists a strictly increasing infinite sequence $n^{\prime}$ such that $\alpha_{n^{\prime}}\left(\tau_{0}\right)$ is negative.

4.2. A system of equations satisfied by the functions $\alpha_{n}$. In this section we obtain a set of differential equations which are satisfied by the functions $\alpha_{n}(\tau)$. These will be used to establish some of the properties of these functions in the remaining of the paper.

Theorem 4.3. The functions $\alpha_{n}: \mathbb{R}^{+} \rightarrow \mathbb{R}$ satisfy the following (infinite) system of differential equations

$$
\frac{\tau}{n} \alpha_{n}^{\prime}(\tau)+\frac{n+1}{n} \alpha_{n}(\tau)=\alpha_{n+1}(\tau), \quad n=1,2, \ldots
$$

Proof. By (3.2) we have that

$$
\begin{aligned}
\alpha_{n+1}(\tau) & =\frac{1}{\tau} \sum_{\rho}\left[1-\left(\frac{\rho}{\rho-\tau}\right)^{n+1}\right] \\
& =\frac{1}{\tau} \sum_{\rho}\left[1-\left(\frac{\rho}{\rho-\tau}\right)^{n}\right]+\frac{1}{\tau} \sum_{\rho}\left(\frac{\rho}{\rho-\tau}\right)^{n}\left[1-\frac{\rho}{\rho-\tau}\right] \\
& =\alpha_{n}(\tau)-\sum_{\rho} \frac{\rho^{n}}{(\rho-\tau)^{n+1}} .
\end{aligned}
$$

On the other hand, differentiating the above expression for $\alpha_{n}$ with respect to $\tau$ gives

$$
\alpha_{n}^{\prime}(\tau)=-\frac{1}{\tau} \alpha_{n}(\tau)-\frac{n}{\tau} \sum_{\rho} \frac{\rho^{n}}{(\rho-\tau)^{n+1}} .
$$

Combining these two expressions yields the desired result.

System (4.1) has a singularity at $\tau=0$ which means that the conditions at this point given by Theorem 2 should be seen as compatibility conditions necessary to ensure that the solutions have a bounded derivative. Thus, in order to determine the solution of this system uniquely, one should add a condition at another point 
which may actually be the behaviour at infinity. In any case, since we are dealing with an infinite system, a more careful analysis would be required to ensure uniqueness under these circumstances. Since for our present purposes the fact that the coefficients $\alpha_{n}$ satisfy (4.1) is sufficient, we will not pursue the matter here.

We also remark that although this system is nonautonomous, it can be transformed into an autonomous system via a change of variables. This is the case if we take, for instance, $\beta_{n}(\tau)=e^{-\tau} \alpha_{n}\left(e^{-\tau}\right)$, yielding

$$
\beta_{n}^{\prime}(\tau)-n \beta_{n}(\tau)=-n \beta_{n+1}(\tau)
$$

The interval of interest is now $[-\log 2,+\infty)$, and we have that

$$
\beta_{n}(-\log 2)=2 \alpha_{n}(2)(\geq 0) \text { and } \lim _{\tau \rightarrow+\infty} \beta_{n}(\tau)=0^{-}
$$

Another approach is to view system (4.1) as a discrete dynamical system defined on the set of $\mathcal{C}^{\infty}(0, \infty)$ functions, with initial condition $\alpha_{1}(\tau)=\xi^{\prime}(\tau) / \xi(\tau)$. In this setting the problem is well-posed, but again we are dealing with a non-autonomous sytem.

4.3. Oscillatory behaviour of the functions $\alpha_{n}$. We shall now study the oscillatory properties of the functions $\alpha_{n}$. We begin by proving the following simple lemma.

Lemma 4.4. For $0 \leq \tau_{1}<\tau_{2}$ we have

$$
\int_{\tau_{1}}^{\tau_{2}} t^{n} \alpha_{n+1}(t) d t=\frac{\tau_{2}^{n+1} \alpha_{n}\left(\tau_{2}\right)-\tau_{1}^{n+1} \alpha_{n}\left(\tau_{1}\right)}{n}, n=1, \ldots
$$

Proof. Multiplying equation (4.1) by $\tau^{n+1}$ we get

$$
\frac{d}{d \tau}\left[\tau^{n+1} \alpha_{n}(\tau)\right]=n \tau^{n} \alpha_{n+1}(\tau)
$$

Integrating now between $\tau_{1}$ and $\tau_{2}$ gives the desired result.

We are now ready to prove Theorem 2

Proceeding as in the proof of Theorem 4.3 and letting $\tau=0$ we get that

$$
\alpha_{n+1}(0)=\alpha_{n}(0)-\sum_{\rho} \frac{1}{\rho}=\alpha_{n}(0)+\frac{\xi^{\prime}(0)}{\xi(0)} .
$$

On the other hand, from the definition of the functions $\alpha_{n}$ we have that $\alpha_{1}(0)=$ $\xi^{\prime}(0) / \xi(0)$ and so $\alpha_{n}(0)=n \xi^{\prime}(0) / \xi(0)$. This proves part (i) of the theorem.

To prove (ii) we argue by induction. Since $\alpha_{1}(\tau)=\xi^{\prime}(\tau) / \xi(\tau)$, we have that $\alpha_{1}(1 / 2)=0$. Letting $\tau_{1}=0$ and $\tau_{2}=1 / 2$ in Lemma 4.4 we obtain that

$$
\int_{0}^{1 / 2} t \alpha_{2}(t) d t=0
$$

and thus $\alpha_{2}$ must have a zero on $(0,1 / 2)$. Assume now that $\alpha_{n}\left(a_{n}\right)=0$ for some $a_{n}$ less than $1 / 2$. Then, letting $\tau_{1}=0$ and $\tau_{2}=a_{n}$ in Lemma 4.4 we obtain that

and the result follows.

$$
\int_{0}^{a_{n}} t^{n} \alpha_{n+1}(t) d t=0
$$

Point (iii) in the theorem follows, in the case of distinct zeros $z_{1}<z_{2}$ of $\alpha_{n}$, in a similar fashion using now Lemma 4.4 with $\tau_{1}=z_{1}$ and $\tau_{2}=z_{2}$. For the case of 
a zero $z$ of multiplicity $m$ of $\alpha_{n}$, it follows by repeated differentiation of (4.1) that $\alpha_{n+1}$ vanishes to order $m-1$ at $z$.

In order to prove point (iv), denote by $\nu_{n}$ the number of zeros of $\alpha_{n}$, counting multiplicities. From points (ii) and (iii) it follows that $\nu_{n} \leq \nu_{n+1}$. We argue by contradiction. If the statement is false, there exists a smallest number $A$ such that $\nu(n) \leq A$ for all $n$. Let $p$ be the smallest number for which $\nu_{p}=A$, and denote the largest zero of $\alpha_{p}$ by $z_{p}$ and its multiplicity by $m$. Then $\alpha_{p+m}$ has all its $A$ positive zeros strictly to the left of $z_{p}$. Denote its largest zero by $z_{p+m}$. Since for $n$ larger than $p+m$ all the zeros of the functions $\alpha_{n}$ must be to the left of $z_{p+m}$, it follows that there is only a finite number of the functions $\alpha_{n}$ which are negative on the interval $\left(z_{m+p}, z_{p}\right)$. Then Proposition 4.1 gives that there are no zeros of $\xi$ in the half-plane $\Re(s)>z_{p+m}$.

On the other hand, we have that the zero at $z_{p}$ of at least one of the functions $\alpha_{p}, \ldots, \alpha_{p+m-1}$ is of odd multiplicity, which means that there is at least one function which takes on negative values on the interval $\left(z_{p+m}, z_{p}\right)$. By Theorem 1 this implies that there must exist zeros of the function $\xi$ with real parts between $z_{p+m}$ and $z_{p}$, giving a contradiction. This concludes the proof of Theorem 2

\section{A FUnCTION DEFINED ON $\mathbb{R}^{2}$}

In this section we extend the definition of the coefficients $\alpha_{n}$ to the case where the parameter $n$ is allowed to vary continuously. This is done by means of one of the expressions for $\alpha_{n}$ used in the previous section, namely, identity (3.2). More precisely, define the function $F: \mathbb{R}^{2} \rightarrow \mathbb{R}$ by

$$
F(x, \tau)=\frac{1}{\tau} \sum_{\rho}\left[1-\left(\frac{\rho}{\rho-\tau}\right)^{x}\right] .
$$

From this and the definition of the coefficients $\alpha_{n}$ it follows that $\alpha_{n}(\tau)=F(n, \tau)$ for $n=1, \ldots$. Many of the properties derived in the previous section also apply to the function $F$. For instance, it is possible to prove in the same way that it will also satify a corresponding system of differential equations, namely,

$$
\frac{\tau}{x} \frac{\partial F(x, \tau)}{\partial \tau}+\frac{x+1}{x} F(x, \tau)=F(x+1, \tau)
$$

It is also clear that $F$ vanishes identically in $\tau$ at $x=0$ and taking derivatives with respect to $x$ and letting $x=0$ yields

$$
\frac{\partial F(0, \tau)}{\partial x}=\frac{1}{\tau} \log [\xi(\tau)],
$$

giving that this derivative is negative for $\tau$ smaller than one and positive for $\tau$ larger than one.

5.1. Alternative representations of the function $F$. There are several ways in which one can transform $F$ in order to obtain other representations of this function. Here we give two which depend on the Taylor coefficients of the logarithmic derivative of $\xi$.

Theorem 5.1. We have that

$$
F(x, \tau)=\sum_{k=0}^{\infty} \frac{\ell_{k}}{(k+1) !} \frac{\Gamma(x+k+1)}{\Gamma(x)} \tau^{k}, \quad|\tau|<R, x \in \mathbb{C},
$$


where $\ell_{k}$ are the Taylor coefficients of the function $\ell(s)=\xi^{\prime}(s) / \xi(s)$ around zero and $R$ is the absolute value of the first zero of the zeta function on the critical line.

Proof. Write

$$
G(x, \tau)=\tau F(x, \tau)=\sum_{\rho}\left[1-\left(\frac{\rho}{\rho-\tau}\right)^{x}\right] .
$$

Then

and

$$
\begin{aligned}
\frac{\partial^{k} G(x, 0)}{\partial \tau^{k}} & =-x(x+1) \ldots(x+k-1) \sum_{\rho} \frac{1}{\rho^{k}} \\
& =x(x+1) \ldots(x+k-1) \frac{1}{(k-1) !} \ell^{(k-1)}(0),
\end{aligned}
$$

$$
G(x, \tau)=\sum_{k=1}^{\infty} \frac{1}{k !} \frac{\Gamma(x+k)}{\Gamma(x)} \ell_{k-1} \tau^{k},
$$

from which the result follows for $F$ upon multiplication by $\tau$ and a change of the summation variable.

The value of the radius of convergence follows from the fact that the only singularities of $F$ (in $\tau$ ) are at the zeros of $\xi$.

From this it follows that at negative integers $F$ is a polynomial in $\tau$-this could also have been obtained by direct inspection of the definition of $F$. More precisely, we have the following

Corollary 5.2. Let $p$ be a positive integer. Then

$$
F(-p, \tau)=\sum_{k=0}^{p-1} \frac{\ell_{k}}{(k+1) !} \prod_{j=0}^{k}(j-p) \tau^{k} .
$$

We also obtain a generalization of an expression given in [C2] for Li's coefficients, which is the sum of a positive and a negative part. To this end, we need to define the coefficients $\eta_{k}$ which appear in the Taylor series development of the logarithmic derivative of $\zeta$ around one. More precisely,

$$
\frac{\zeta^{\prime}(s)}{\zeta(s)}=-\frac{1}{s-1}-\sum_{k=0}^{\infty} \eta_{k}(s-1)^{k}
$$

These coefficients may also be written in terms of the von Mangoldt function see [C2], for instance.

Corollary 5.3. Define

$$
P(x, \tau)=\sum_{k=1}^{\infty} \frac{1}{(k+1) !} \frac{\Gamma(x+k+1)}{\Gamma(x)}\left[\left(1-2^{(-k-1)}\right) \zeta(k+1)-1\right] \tau^{k}
$$

and

$$
N(x, \tau)=\left[\log (2 \sqrt{\pi})-1-\frac{\gamma}{2}\right] x+\sum_{k=1}^{\infty} \frac{(-1)^{k}}{(k+1) !} \frac{\Gamma(x+k+1)}{\Gamma(x)} \eta_{k} \tau^{k} .
$$

Then, for positive $x$ and $\tau$, the functions $P$ and $N$ are positive and negative, respectively, and

$$
F(x, \tau)=P(x, \tau)+N(x, \tau) .
$$


Remark 5.4. This result generalizes one of the expressions for Li's coefficients given in [C2], while explicitly separating the positive and negative parts of the expansion.

Proof. We have that

$$
\log [\xi(s)]=\log \left(\frac{s}{2}\right)-\frac{s}{2} \log (\pi)+\log \left[\Gamma\left(\frac{s}{2}\right)\right]-\sum_{k=1}^{\infty} \frac{\eta_{k-1}}{k}(s-1)^{k},
$$

from which it follows upon exchanging $s$ with $1-s$ and differentiating with respect to $s$ that

$$
\ell(s)=-\frac{1}{1-s}+\log (\sqrt{\pi})-\frac{1}{2} \psi\left(\frac{1-s}{2}\right)+\sum_{k=0}^{\infty}(-1)^{k} \eta_{k} s^{k},
$$

where $\psi$ is the digamma function defined by $\psi(s)=[\log (\Gamma(s))]^{\prime}$. Since

$$
-\frac{1}{2} \psi\left(\frac{1-s}{2}\right)=\frac{\gamma}{2}+\log (2)+\sum_{k=1}^{\infty}\left(1-2^{-k-1}\right) \zeta(k+1) s^{k},
$$

we are finally led to

$$
\ell(s)=\log (2 \sqrt{\pi})-1-\frac{\gamma}{2}+\sum_{k=1}^{\infty}\left[-1+\left(1-2^{-k-1}\right) \zeta(k+1)+(-1)^{k} \eta_{k}\right] s^{k} .
$$

This last expression gives the coefficients $\ell_{k}$ used in Theorem 5.1 from which the expression for $F$ in terms of $P$ and $N$ follows. To prove that $P$ and $N$ are positive end negative, respectively, it is sufficient to note that $\left(1-2^{(-k-1)}\right) \zeta(k+1)-1$ is positive, and that the coefficients $\eta_{k}$ are positive for odd $k$ and negative for even $k$. The first of these statements follows directly from the fact that $\zeta(k+1)$ is larger than $1+2^{-(k+1)}+3^{-(k+1)}$, while the second can be found in $[\mathrm{C} 2$.

We shall now show the existence of a region close to the $x$ axis where the function $F$ remains negative. In particular, this will imply that the sequence $a_{n}$ referred to in Theorem 22 if it goes to zero, cannot do so faster than $c / n$, for some positive constant $c$.

Theorem 5.5. There exist positive constants $\tau_{0}$ and $x_{0}$ such that $F$ takes on negative values on the set

$$
X=\left\{(x, \tau) \in \mathbb{R}^{2}: x_{0}<x<\tau_{0} / \tau\right\} .
$$

Proof. From Theorem [5.1] we have that

$$
r F\left(\frac{1}{r}, r \tau\right)=\sum_{k=0}^{\infty} \frac{\ell_{k}}{(k+1) !}(1+r) \ldots(1+k r) \tau^{k}
$$

and thus

$$
\lim _{r \rightarrow 0}\left[r F\left(\frac{1}{r}, r \tau\right)\right]=\sum_{k=0}^{\infty} \frac{\ell_{k}}{(k+1) !} \tau^{k} .
$$

Since $\ell_{0}$ is negative, there exists a positive number $\tau_{0}$ such that the function appearing on the right-hand side of (5.1) is negative at $\tau_{0}$. Hence, for positive $r$ sufficiently small, $F(1 / r, r t)$ must be negative for $0<t<\tau_{0}$. To obtain the result, write $x=1 / r$ and $\tau=r t$. 
The above result does not show if the region close to the $x$-axis thins out as $x$ goes to infinity, or if there is a strip where $F$ is negative. In fact, we conjecture that the latter situation does not occur, and thus that the sequence $a_{n}$ in Theorem 2 does indeed go to zero.

If we use the binomial series development for the power term in the definition of $F$ we get a slightly different expression.

Theorem 5.6. We have that

$$
F(x, \tau)=\sum_{k=0}^{\infty} \frac{\Gamma(x+1)}{(k+1) ! \Gamma(x-k)} \ell_{k}(\tau) \tau^{k}
$$

where

$$
\ell_{k}(\tau)=\frac{1}{k !} \frac{d^{k}}{d \tau^{k}}[\ell(\tau)]
$$

Proof.

$$
\begin{aligned}
F(x, \tau) & =\frac{1}{\tau} \sum_{\rho}\left[1-\left(\frac{\rho}{\rho-\tau}\right)^{x}\right] \\
& =\frac{1}{\tau} \sum_{\rho}\left[1-\left(1+\frac{\tau}{\rho-\tau}\right)^{x}\right] \\
& =\frac{1}{\tau} \sum_{\rho}\left[1-\sum_{k=0}^{\infty} \frac{\Gamma(x+1)}{k ! \Gamma(x+1-k)}\left(\frac{\tau}{\rho-\tau}\right)^{k}\right] \\
& =-\sum_{k=1}^{\infty} \frac{\Gamma(x+1)}{k ! \Gamma(x+1-k)} \tau^{k-1} \sum_{\rho} \frac{1}{(\rho-\tau)^{k}} \\
& =\sum_{k=1}^{\infty} \frac{\Gamma(x+1)}{k ! \Gamma(x+1-k)} \frac{\tau^{k-1}}{(k-1) !} \frac{d^{k-1}}{d \tau^{k-1}}[\ell(\tau)],
\end{aligned}
$$

which gives the desired result.

\section{Concluding REMARKS}

As was pointed out in the Introduction, the criterion given here is not restricted to the $\xi$ function, and most of the results presented may be extended to a fairly general class of complex functions. In particular, we mention that the set of equations (4.1) satisfied by the coefficients $\alpha_{n}$ (and the function $F$ ) is independent of the function under study, and that the sequence $\alpha_{n}$ will be determined by the function $\alpha_{1}$, for instance. The alternative representations for the function $F$ given in Section [5] also apply to this more general case, if we replace the Taylor coefficients of the function $\ell$ by those of the logarithmic derivative of a function $f$. In relation to this, we stress again that for a function $f$ of the form (1.4) it would also have been possible to use the more general approach of Bombieri and Lagarias BL throughout the paper, in which case it would not have been necessary to require the non-negativity condition on the Taylor coefficients of $f$.

Regarding the specific case of the function $\xi$, we note that for $z_{0}$ in $(-1,0)$ the disk $\left|z-z_{0}\right|<1-z_{0}$ contains the closure of the unit disk centred at zero, with the exception of the point $z=1$. Since $\varphi$ vanishes at the points on the unit circle which correspond to the nontrivial zeros of $\zeta$ on the critical line, $\psi$ cannot be analytic 
on the larger circle which implies that the coefficients $d_{n}\left(z_{0}\right)$ must then take on negative values when $z_{0}$ is negative, and hence when $\tau$ is less than one we have the same type of behaviour for the coefficients $\alpha_{n}$.

On the other hand, and as was also pointed out in the Introduction, for $\tau$ greater than or equal to two, the half-plane $\Re(z)<\tau / 2$ contains the whole of the critical strip, and hence the $\alpha_{n}$ 's will be non-negative in this case.

Besides giving a criterion for the existence/nonexistence of zeros in half-planes, we hope that the results presented here will also help to provide some insight into the behaviour of the coefficients $\lambda_{n}$. In particular, it might be interesting to analyse numerically the situation where $\tau$ is close to but less than one, and see how the negative part of the sequence $\alpha_{n}$ behaves as $\tau$ approaches one. Note also that when $\tau$ is zero, all functions $\alpha_{n}$ (with positive $n$ ) are negative.

Acknowledgments. I would like to thank Mark Coffey for having made available a preliminary version of $\mathrm{C} 2$.

\section{REFERENCES}

[BL] E. Bombieri and J. C. Lagarias, Complements to Li's criterion for the Riemann hypothesis, J. Number Theory 77 (1999), 274-287.

[B] F. C. S. Brown, Li's criterion and zero-free regions of $L$-functions, J. Number Theory 111 (2005), 1-32.

[C1] M. W. Coffey, Relations and positivity results for the derivatives of the Riemann $\xi$ function, J. Comput. Appl. Math. 166 (2004), 525-534.

[C2] M. W. Coffey, New results on the Li constants, preprint 2004.

[E] H. M. Edwards, "Riemann's Zeta Function," Academic Press, New York, 1974.

[K] J. B. Keiper, Power series expansions of Riemann's $\xi$ function, Math. Comp. 58 (1992), $765-773$

[K] K. Knopp, Theory and application of infinite series, Dover, New York, 1990.

[L] J. C. Lagarias, Li coefficients for automorphic $L$-functions, arXiv: math.NT/0404394 v3.

[L] X.-J. Li, The positivity of a sequence of numbers and the Riemann hypothesis, J. Number Theory 65 (1997), 325-333.

[M1] K. Maslanka, An effective method of computing Li's coefficients and their unexpected properties, arXiv: math.NT/0402168 v2.

[M2] K. Maslanka, An explicit formula relating Stieljes constants and Li's numbers, arXiv: math.NT/0406312 v2.

[P] L. D. Pustyl'nikov, On a property of the classical zeta function associated with the Riemann conjecture on zeros (Russian), Uspekhi Mat. Nauk 54 (1999), 259-260; translation in Russian Math. Surveys 54 (1999), 262-263.

[V] A. Voros, A sharpening of Li's criterion for the Riemann hypothesis, arXiv math.NT/0404213

Departamento de Matemática, Instituto Superior Técnico, Av.Rovisco Pais, 1049-001 Lisboa, Portugal.

E-mail address: pfreitas@math.ist.utl.pt 